> Dans la terminaison nerveuse, l'arrivée d'un potentiel d'action induit la libération du neuromédiateur dans l'espace synaptique. Cette libération est la dernière étape d'une série de réactions aboutissant à la fusion de la membrane de la vésicule synaptique à celle de membrane plasmique, réactions impliquant un grand nombre de protéines. Parmi celles-ci, l'ATPase à protons de type vacuolaire (V-ATPase) joue un rôle essentiel à un double niveau, lors du stockage des neuromédiateurs dans les vésicules synaptiques d'abord, lors de l'étape ultime de leur libération ensuite. <

\section{La libération des neuromédiateurs : le double jeu de la V-ATPase}

\section{Nicolas Morel, Jean-Marc Philippe}

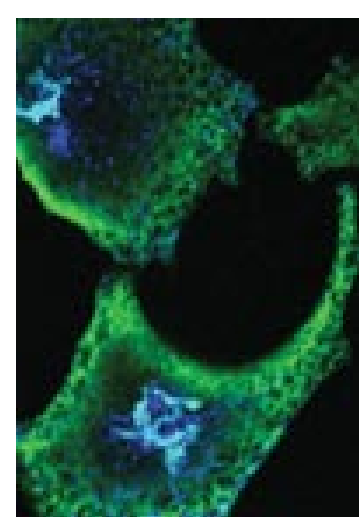

Laboratoire de Neurobiologie Cellulaire et Moléculaire, Cnrs UPR 9040, 91198 Gif-sur-Yvette, France. nicolas.morel@nbcm.cnrs-gif.fr
Les neuromédiateurs sont synthétisés et stockés dans des régions spécialisées du neurone, les terminaisons nerveuses, situées à l'extrémité du prolongement axonal, à distance du corps cellulaire. Ils sont libérés dans l'espace synaptique, au contact de la cellule cible, au niveau de domaines particuliers de la membrane pré-synaptique, les zones actives. Celles-ci sont caractérisées par une accumulation de canaux calciques dépendant du voltage et par la présence de vésicules synaptiques amarrées à la membrane [1]. L'arrivée d'un potentiel d'action dépolarise la terminaison nerveuse, induisant l'ouverture de ces canaux calciques et une augmentation brutale de la concentration cytosolique de calcium qui peut atteindre, dans des microdomaines situés sous les zones actives, des valeurs très élevées (200 $\mu \mathrm{m}$ et plus) [2]. Le neuromédiateur est alors libéré moins de $200 \mu$ s après l'activation des canaux calciques [3]. Cette libération, très rapide et dépendante du calcium, est la dernière étape d'une série de réactions très similaires à celles qui interviennent lors d'événements de fusion membranaire beaucoup plus lents, impliquant des protéines conservées de la levure à l'homme [4]. Nous décrirons le double jeu de l'une de ces protéines, I'ATPase à protons de type vacuolaire (V-ATPase), qui intervient dans le stockage des neuromédiateurs dans les vésicules synaptiques et lors de leur libération.

\section{V-ATPase et stockage des neuromédiateurs}

La V-ATPase transporte des protons à travers la membrane de divers organites intracellulaires acides (lysosomes, citernes du trans-Golgi, endosomes, 
granules de sécrétion, ...). Cette activité, indispensable à la survie cellulaire, intervient pendant la maturation et la dégradation des protéines, lors de l'endocytose de divers récepteurs et au cours de divers transports membranaires intracellulaires. La V-ATPase est une enzyme multimérique de grande taille, organisée en deux domaines, Vl et V0 (Figure 1). Le domaine cytoplasmique Vl $(500 \mathrm{kDa})$ est constitué par huit $(\rightarrow) \mathrm{m} / \mathbf{s} \quad$ sous-unités différentes (stœchiométrie $A 3, B 3, \mathrm{Cl}$, 2001, $\left.\mathbf{n}^{\circ} \mathbf{5}, \quad D l, \varepsilon l, F l, G l, H l\right)$, et hydrolyse l'ATP [5]. Le domaine p. 669 membranaire $V 0$ est un complexe de $250 \mathrm{kDa}$ comprenant au moins trois sous-unités (stœchiométrie al, dl, c6). La sous-unité c est organisée en une couronne protéolipidique responsable du transport des protons. L'hydrolyse de I'ATP par les trois sous-unités A de VI entraîne la rotation dans la membrane de cette couronne de protéolipides et la translocation des protons liés à sa périphérie [5]. Les domaines Vl et VO de la VATPase sont synthétisés séparément et s'associent secondairement pour former l'enzyme active. Leur association est réversible, cette réversibilité étant I'un des mécanismes de régulation de l'activité enzymatique [5]. Dans les neurones, les domaines VI et VO sont transportés à des vitesses différentes dans les axones et ne s'assemblent qu'une fois arrivés dans les terminaisons nerveuses [6].

Les vésicules synaptiques possèdent une V-ATPase [7] qui accumule des protons dans ces organites (Figure 2). Ce transport est électrogène - créant un double gradient de potentiel et de $\mathrm{pH}$ de part et d'autre de la membrane vésiculaire -, de potentiel positif et de $\mathrm{pH}$ acide $(5,2)$ dans la vésicule [8]. Ce gradient électrochimique de protons est secondairement utilisé par des transporteurs vésiculaires, spécifiques de chacun des neuromédiateurs, pour concentrer ceux-ci dans les vésicules synaptiques [9]. Les concentrations de neuromédiateurs atteintes sont très élevées, supérieures à $500 \mathrm{~mm}$ d'acétylcholine, dans le cas, par exemple, des vésicules cholinergiques [10].

Les vésicules synaptiques ne constituent pas une population homogène dans la terminaison nerveuse. Une sous-population de vésicules (5 à $10 \%$ ), probablement amarrées aux zones actives, est préférentiellement utilisée pour libérer le neuromédiateur lors de l'activité synaptique [11-13]. Une fois vidées de leur contenu, ces vésicules se remplissent à nouveau avec le neuromédiateur synthétisé dans le cytoplasme, probablement sans quitter la zone active. Elles sont réutilisées plusieurs fois sans se mélanger à la population des vésicules synaptiques de réserve $[11,13,14]$. La libération de neuromédiateurs est quantique et la taille des paquets de neuromédiateurs libérés est constante [15]. Si le quantum correspond au contenu d'une vésicule synaptique, il faut postuler que le mécanisme de libération choisit, parmi les vésicules synaptiques de la zone active, celles qui sont pleines de neuromédiateur et ignore celles qui sont en cours de remplissage.

\section{Libération des neuromédiateurs via un pore de fusion?}

Le rôle des protéines SNARE lors de la fusion membranaire est bien documenté $[4,16,17](\rightarrow)$. Ces protéines permettent l'amarrage des membranes grâce à la formation de complexes très stables associant v-SNARE (VAMP/synaptobrévine 2 dans la membrane des vésicules synaptiques) et t-SNARE (syntaxine 1 et SNAP25 dans la membrane présynaptique) (Figure 3, étape 1). L'augmentation de la concentration cytosolique de calcium induit ensuite l'ouverture d'un pore de fusion (Figure 3, étape 2) [18]. Ce pore de fusion peut ensuite, soit se refermer après un délai variable (Figure 3, étape 4), soit évoluer vers une fusion membranaire (Figure 3, étape 3). Dans le premier cas, l'ouverture transitoire du pore est suffisante pour permettre la libération du neuromédiateur contenu dans une petite vésicule synaptique [19] mais insuffisante pour laisser passer des peptides de granules de plus grande taille. La vésicule vide est alors immédiatement disponible pour pomper les protons puis le neuromédiateur (Figure 3, étapes 6 et 7). Dans le second cas, la taille du pore de fusion augmente progressivement, évoluant jusqu'à la fusion complète des membranes et la libération totale du contenu vésiculaire (Figure 3, étape 3). L'évolution du pore de fusion - ouverture transitoire ou expansion et fusion membranaire - semble dépendre de son environnement. Les événements d'ouverture transitoire sont prépondérants à très fortes concentrations de

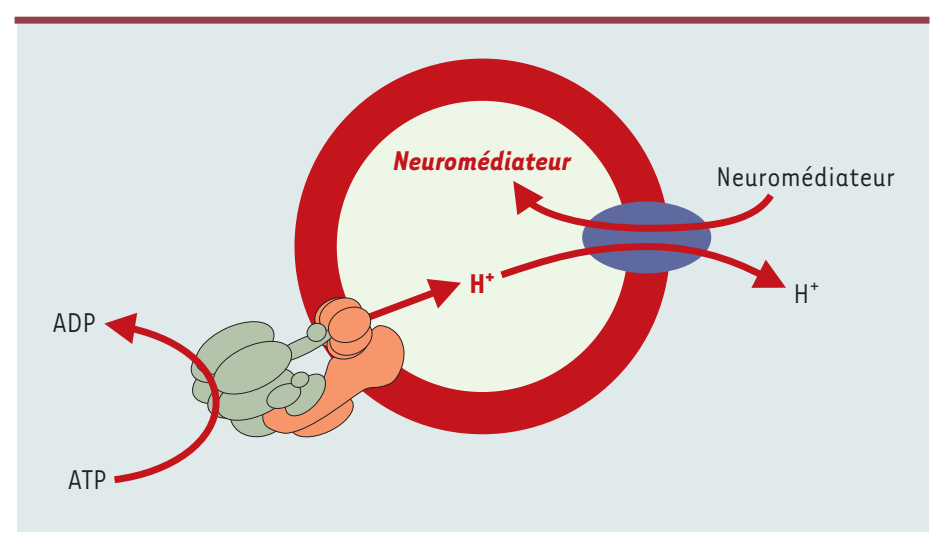

Figure 2. Mécanisme de transport des neuromédiateurs dans les vésicules synaptiques. La V-ATPase accumule des protons $\left(\mathrm{H}^{+}\right)$dans les vésicules synaptiques. Le gradient électrochimique de protons ainsi créé est utilisé par un transporteur vésiculaire (bleu), qui confère à chaque type de vésicules sa spécificité pour un neuromédiateur (acétylcholine, glutamate, monoamines, ...). 
calcium (conditions qui ne sont rencontrées que dans la zone active lors de l'activité synaptique) et minoritaires pour des concentrations plus faibles [20]. L'expansion du pore de fusion et l'exocytose avec fusion membranaire sont inhibées par la staurosporine, inhibiteur de la protéine kinase C [21-23], alors que la libération de médiateur n'est pas diminuée [21]. Il est donc probable que, lors de la stimulation, c'est l'ouverture transitoire du pore de fusion qui permet la libération du neuromédiateur contenu dans les vésicules synaptiques amarrées à la zone active. La nature lipidique ou protéique de ce pore est encore controversée [16, 17, 24].

\section{V-ATPase et pore de fusion}

Trois types d'arguments expérimentaux suggèrent que les sous-unités protéolipidiques de la V-ATPase peuvent for-

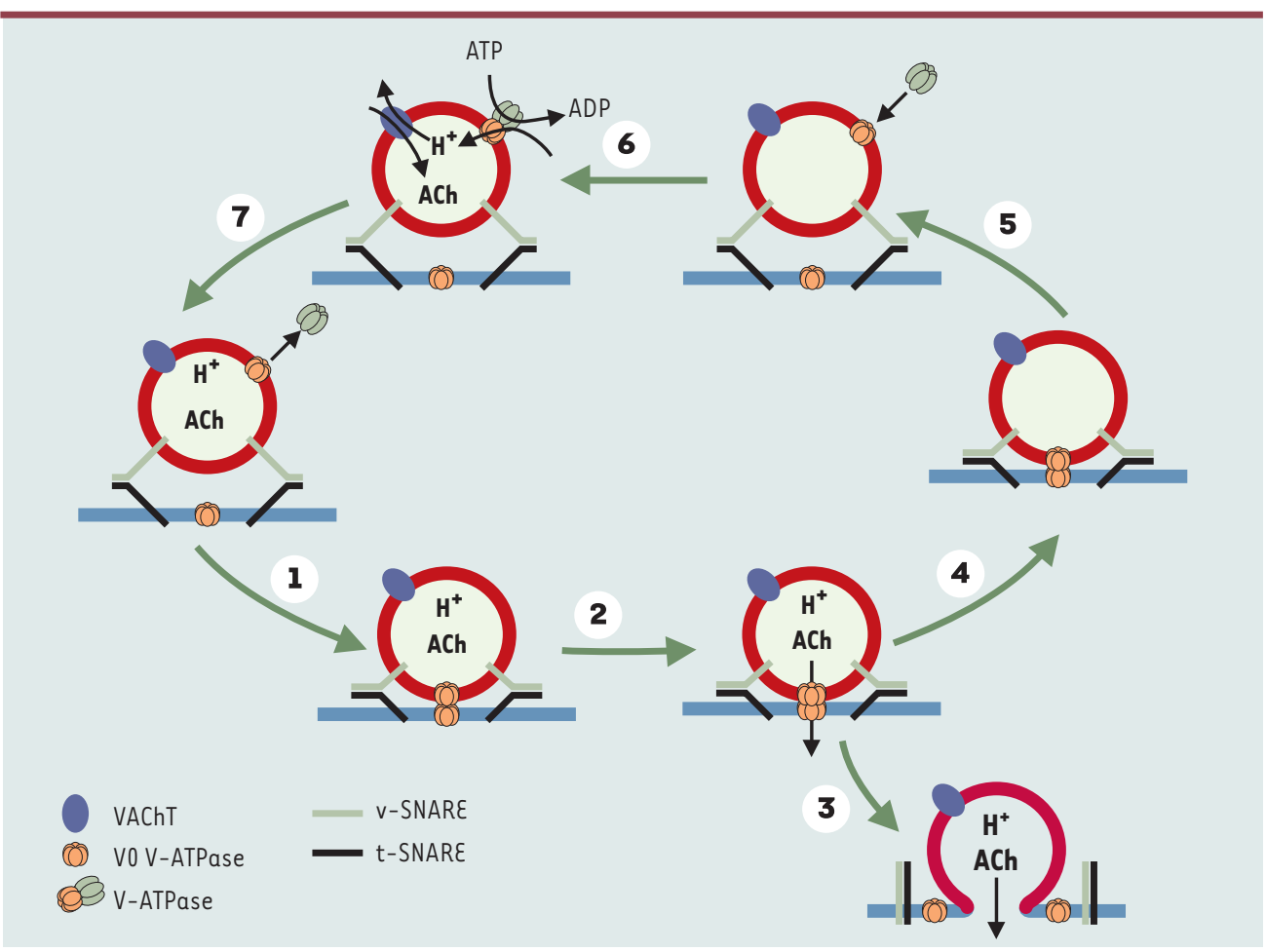

Figure 3. V-ATPase et libération de neuromédiateur (ici l'acétylcholine). La formation des complexes SNARE ancre la vésicule synaptique à la membrane présynaptique (étape 1). Cela permet la formation d'un trans-complexe V0V0 constituant le pore de fusion. L'élévation de la concentration cytosolique de calcium induit ensuite l'ouverture de ce pore (étape 2). Le pore de fusion peut se dilater et évoluer vers une fusion membranaire complète (étape 3). Il peut aussi se refermer après un délai variable (étape 4); son ouverture transitoire est suffisante pour permettre la libération du neuromédiateur contenu dans la vésicule synaptique. Ensuite, les complexes SNARE et le pore de fusion se dissocient (étape 5); le domaine catalytique Vl peut alors se fixer au domaine V0 ainsi libéré. Cela restaure l'activité de la V-ATPase et la vésicule se remplit de neuromédiateur (étape 6). Une fois la vésicule pleine, le gradient électrochimique de protons n'est plus utilisé par le transporteur et atteint son maximum. Cela induit le détachement du domaine VI (étape 7), démasquant le domaine V0 qui devient disponible pour reformer le pore de fusion (étape 1). VAChT: transporteur acétylcholine. mer un tel pore. Le premier repose sur des expériences de reconstitution fonctionnelle dans des membranes artificielles: après incorporation dans la membrane artificielle de liposomes remplis d'acétylcholine, un homo-oligomère de la sous-unité protéolipidique c de la V-ATPase purifiée à partir de terminaisons nerveuses de torpille (médiatophore) est capable de libérer l'acétylcholine sous l'action du calcium [25, 27]. De même, ce protéolipide - extrait à partir de V-ATPase de levure et reconstitué dans une membrane artificielle - forme un pore qui s'ouvre en présence de calcium et de calmoduline [28].

Un deuxième argument repose sur des expériences de transfection fonctionnelle: des cellules de neuroblastome, transfectées par des plasmides dirigeant la synthèse de la sous-unité $c$ de torpille, acquièrent la capacité de libérer l'acétylcholine de manière quantique et dépendante du calcium [26]. Enfin, des expériences de fusion homotypique des vacuoles de levure mettent en évidence, après l'amarrage, une étape dépendante du calcium et de la calmoduline [4]. Peters et al [28] ont montré que ce sont les protéolipides du domaine VO de la V-ATPase qui interagissent avec la calmoduline. Lors de la fusion de deux vacuoles, un trans-complexe V0-V0 s'assemble par apposition de deux domaines vo mis en contact par les protéines SNARE. Le trans-complexe VO-VO constituerait alors, au niveau du site de fusion, un canal traversant les deux membranes [28]. L'hydrophobicité de la couronne de protéolipides constituant la paroi de ce canal apparaît favorable à une éventuelle expansion radiale du pore par interposition de lipides membranaires [24].

Ainsi, le domaine V0 de la V-ATPase pourrait constituer le pore de fusion impliqué dans les fusions membranaires [16, 17, 23, $24,28]$ et dans la libération de neuromédiateur [27]. Le même édifice protéique remplirait deux 
fonctions très différentes, transporter des protons lorsqu'il est associé au domaine catalytique V1, ou constituer un pore de grande conductance lorsqu'il est dans un trans-complexe V0-V0. La sous-unité protéolipidique c étant très conservée de la levure à l'homme, et aucune isoforme de c n'ayant été décrite chez les vertébrés [5], des contraintes fonctionnelles importantes ont dû s'opposer au cours de l'évolution à une spécialisation divergente des protéolipides impliqués dans ces deux types de complexes (V0-Vl et V0-V0).

L'utilisation du même domaine Vo de la vésicule synaptique, successivement associé à VI dans la V-ATPase active, puis à un autre $V 0$ dans le pore de fusion, permet l'émergence d'une caractéristique fonctionnelle nouvelle, illustrée dans la Figure 3. Lorsque la V-ATPase est active (Figure 3, étape 6), elle produit un gradient de protons qui est utilisé pour accumuler le neuromédiateur (ici l'acétylcholine) dans la vésicule synaptique. Lorsque celle-ci est pleine d'acétylcholine, le transporteur vésiculaire (VAChT) n'utilise plus le gradient électrochimique de protons (gradient de $\mathrm{pH}$ et/ou gradient de potentiel), gradient qui peut alors atteindre sa valeur maximale. Nous proposons que la V-ATPase se dissocie alors (Figure 3, étape 7). La dissociation de Vl est un des moyens de régulation physiologique de l'activité de la V-ATPase [5]. Des acides aminés de la sous-unité a de V0, situés dans la lumière vacuolaire, contrôleraient l'assemblage du domaine cytoplasmique Vl au domaine membranaire V0 [29]. La dissociation de la V-ATPase expose le domaine V0, uniquement si le gradient électrochimique de protons est maximal lorsque la vésicule est pleine de médiateur. Ce domaine VO peut maintenant interagir avec un autre V0 présent dans la membrane présynaptique pour former le pore de fusion [27] (Figure 3, étape 1). Ainsi, seules des vésicules pleines sont utilisées pour libérer le neuromédiateur, rendant compte du caractère quantique de la libération. Un tel mécanisme est transposable à tout événement intervenant dans le trafic membranaire en impliquant des compartiments acides.

La V-ATPase pourrait donc intervenir à plusieurs étapes lors des processus de fusion membranaire et lors de la libération des neuromédiateurs, et son double jeu pourrait expliquer le caractère quantique de la libération des neuromédiateurs. $\diamond$

\section{TIRÉS À PART}

N. Morel

\section{SUMMARY}

Neurotransmitter release: the dark side of the V-ATPase Neurotransmitters are synthesized in the cytoplasm of nerve terminals and stored in synaptic vesicles. Within a msec after stimulation, they are released from synaptic vesicles that are docked to the nerve terminal membrane. Neurotransmitters flow through a fusion pore that forms across the two interacting membranes and then expends, allowing fusion of the synaptic vesicle membrane within the presynaptic membrane. Vacuolar-type proton ATPase (V-ATPase) is essential for neurotransmitter storage. It accumulates protons within synaptic vesicles, generating an electrochemical gradient used by specific transporters to concentrate neurotransmitters. V-ATPase is a complex enzyme made of a catalytic cytoplasmic domain that hydolyses ATP, and a VO membrane domain which translocates protons. We review data that also implicate the VO membrane domain in the formation of the fusion pore. Therefore, the same Vo membrane domain of synaptic vesicle V-ATPase could successively translocate protons, when associated to the catalytic domain, and form the fusion pore, when associated in a trans-complex with a presynaptic membrane V0 domain. Functional implications for quantal transmitter release are discussed. $\bullet$

\section{RÉFÉRENCES}

1. Harlow ML, Ress D, Stoschek A, Marshall RM, McMahan UJ. The architecture of the active zone material at the frog's neuromuscular junction. Nature 2001; 409: 479-84.

2. Llinas R, Sugimori M, Silver RB. Microdomains of high calcium concentration in a presynaptic terminal. Science 1992; 256: 677-9.

3. Llinas R, Steinberg IZ, Walton K. Relationship between presynaptic calcium current and postsynaptic potential in squid giant synapse. Biophys J 1981; 33: 323-51.

4. Wickner W, Haas A. Yeast homotypic vacuole fusion: a window on organelle trafficking mechanisms. Annu Rev Biochem 2000; 69: 247-75.

5. Nelson N, Harvey WR. Vacuolar and plasma membrane proton-adenosine triphosphatases. Physiol Rev 1999; 79: 361-85.

6. Morel N, Gérard V, Shiff G. Vacuolar $\mathrm{H}^{+}$ATPase domains are transported separately in axons and assemble in Torpedo nerve endings. J Neurochem 1998; 71: 1702-8.

7. Yamagata SK, Parsons SM. Cholinergic synaptic vesicles contain a V-type and a Ptype ATPase. J Neurochem 1989; 53: 1354-62.

8. Michaelson DM, Angel I. Determination of $\Delta \mathrm{pH}$ in cholinergic synaptic vesicles: its effects on storage and release of acetylcholine. Life Sci 1980 : 27: 39-44.

9. Varoqui H, Erickson JD. Vesicular neurotransmitter transporters. Mol Neurobiol 1997; 15: 165-92.

10. Ohsawa K, Dowe GHC, Morris SJ, Whittaker VP. The lipid and protein content of cholinergic synaptic vesicles from the electric organ of 
Torpedo marmorata purified to constant composition: implication for vesicular structure. Brain Res 1979; 161: 447-57.

11. Suskiw JB, Zimmermann $H$, Whittaker VP. Vesicular storage and release of acetylcholine in Torpedo electroplaque synapses. J Neurochem 1978; 30: 1269-80.

12. Koenig JH, Ikeda K. Contribution of active zone subpopulation of vesicles to evoked and spontaneous release. J Neurophysiol 1999; 81: 1495-505.

13. Südhof TC. The synaptic vesicle cycle revisited. Neuron 2000; 28 : 317-20.

14. Pyle JL, Kavalali हT, PiedrasRenteria ES, Tsien RW. Rapid reuse of readily releasable pool vesicles at hippocampal synapses. Neuron 2000; 28 : 221-31.

15. Van der Kloot W, Molgo J. Quantal acetylcholine release at the vertebrate neuromuscular junction. Physiol Rev 1994; 74: 899-991.
16. Mayer A. What drives membrane fusion in eukaryotes? Trends Biochem Sci 2001; 26: 717-23.

17. Bruns D, Jahn R. Molecular determinants of exocytosis. Pflug Arch 2002 ; 443: 333-8.

18. Lindau M, Almers W. Structure and function of fusion pores in exocytosis and ectoplasmic membrane fusion. Curr Opin Cell Biol 1995; 7 : 509-17.

19. Alvarez de Toledo G, Fernandez-Chacon R, Fernandez JM. Release of secretory products during transient vesicle fusion. Nat 1993; 363: 554-8.

20. Ales $\varepsilon$, Tabares L, Poyato JM, Valero V, Lindau $M$, Alvarez de Toledo G. High calcium concentrations shift the mode of exocytosis to the «kiss and run» mechanism. Nat Cell Biol 1999; $1:$ 40-4.

21. Henkel AW, Betz WJ. Staurosporine blocks evoked release of FMl-43 but not acetylcholine from frog motor nerve terminals.

J Neurosci 1995; 15: 8246-58.

22. Scepek S, Coorssen JR, Lindau M. Fusion pore expansion in horse eosinophils is modulated by $\mathrm{Ca}^{2+}$ and protein kinase $\mathrm{C}$ via distinct mechanisms. EMBO J 1998; 17: 4340-5.

23. Henkel AW, Kang G, Kornhuber J. A common molecular machinery for exocytosis and the "kiss and run" mechanism in chromaffin cells is controlled by phosphorylation. J Cell Sci 2001; 114: 4613-20.

24. Zimmerberg J. How can proteolipids be central players in membrane fusion? Trends Cell Biol 2001; 11 : 233-35.

25. Israël M, Morel N, Lesbats B, Birman S, Manaranche R. Purification of a presynaptic protein that mediates a calcium dependent translocation of acetylcholine. Proc Natl
Acad Sci USA 1986; 83:

9226-30.

26. Falk-Vairant J, Corrèges $P$, Eder-Colli L, et al. Quantal acetylcholine release induced by mediatophore transfection. Proc Natl Acad Sci USA 1996; 93: 5203-7.

27. Morel N, Dunant $Y$, Israël $M$. Neurotransmitter release through the $\mathrm{V} 0$ sector of $\mathrm{V}$ ATPase. J Neurochem 2001; 79: 485-8.

28. Peters C, Bayer MJ, Bühler $S$, Andersen JS, Mann M, Mayer A. Trans-complex formation by proteolipid channels in the terminal phase of membrane fusion. Nature 2001; 409: 581-8.

29. Leng XH, Nishi T, Forgac $M$. Transmembrane topography of the $100 \mathrm{kDa}$ a subunit (Vphlp) of the yeast vacuolar proton-translocating ATPase. J Biol Chem 1999; 274: 14655-61.

\section{Une technologie de pointe pour une recherche de pointe Microarray Systems}

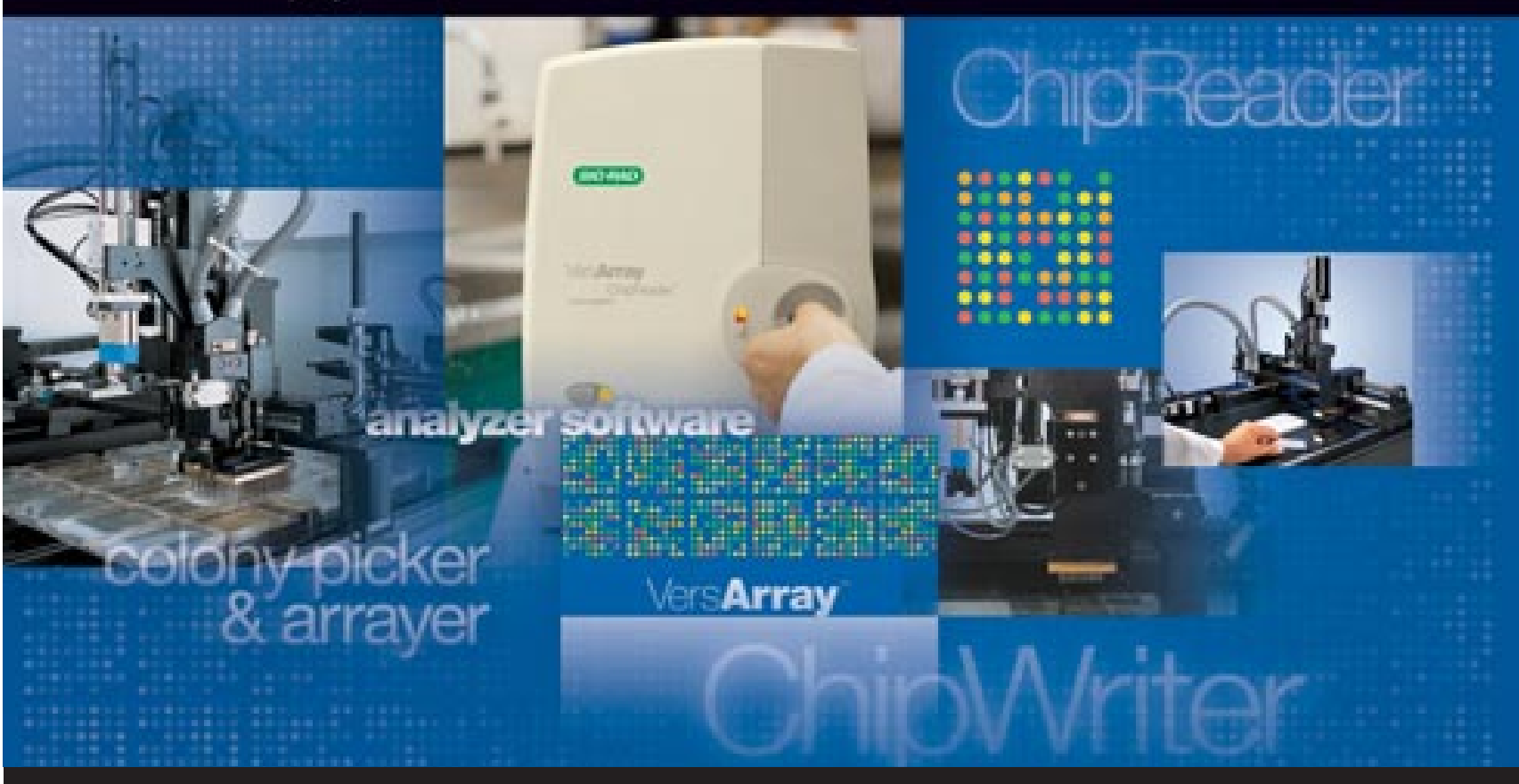

